\title{
Pulse article: How do you do the international standards for neurological classification of SCI anorectal exam?
}

\author{
Marcalee Alexander ${ }^{1,2,3} \cdot$ Hammad Aslam² $\cdot$ Ralph J. Marino $\oplus^{4}$ \\ Received: 26 July 2017 / Revised: 13 September 2017 / Accepted: 18 September 2017 \\ (C) International Spinal Cord Society 2017
}

\begin{abstract}
Study Design Online survey of spinal cord injury (SCI) practitioners.
\end{abstract}

Objectives Determine provider understanding and routine performance of International Standards for Neurologic Classification of Spinal Cord Injury (ISNCSCI) anorectal examination.

Setting International online questionnaire.

Methods A descriptive survey was developed to assess current performance of the ISNCSCI anorectal exam. Information about this survey was disseminated through social email and international societies between the months of March and April 2017.

Results Two hundred and fifteen SCI practitioners completed the survey. Of these, 157 (73\%) were specialists in physical medicine and rehabilitation, $32(15 \%)$ were physical therapists, 6 were neurologists, 6 were orthopedists, and 3 were neurosurgeons. Of responders, $90 \%$ routinely personally performed the anal exam of the ISNCSCI, of whom, 42 placed firm pressure against the anal sphincter, 58 placed firm pressure against the rectal wall, and 87 placed firm pressure against the anal sphincter and rectal wall as part of the exam. On a routine basis, $74 \%$ tested sensation at the junction of the anal sphincter and skin, $82 \%$ had the patient attempt to squeeze their finger, and $50 \%$ performed the bulbocavernosus reflex.

Conclusions Marked inconsistency exists in the performance of the ISNCSCI anal examination. Researchers and clinicians need more education about the performance of the examination. Consideration should be given for the components of deep rectal sensation and anal pressure to be separately documented in the neurologic exam and for researchers to focus separately on recovery of sacral function vs. ambulatory function in clinical trials.

\section{Introduction}

The most established aspect of the care of persons with spinal cord injury (SCI) may be the need for documentation

Marcalee Alexander

spinalcordmd@live.com

1 Birmingham VA Medical Center, 700 South 19th Street, Birmingham, AL 35233, USA

2 Department of Physical Medicine and Rehabilitation, University of Alabama School of Medicine, Birmingham, AL, USA

3 Department of Physical Medicine and Rehabilitation, Harvard School of Medicine, Boston, MA, USA

4 Department of Rehabilitation Medicine, Sidney Kimmel Medical College at Thomas Jefferson University, Philadelphia, PA, USA via the International Standards for Neurologic Classification of Spinal Cord Injury (ISNCSCI) [1-5]. These standards were first developed as the Standards for Neurological Classification of Spinal Cord Injury Patients in 1982 [6] and have subsequently undergone multiple revisions with the most notable being a change in the definition of incomplete SCI in 1992 [7] to be based upon the presence of sacral sparing rather than preservation of motor and sensory function greater than 3 levels below the injury.

While the original standards [6] were primarily developed as a clinical tool, as the performance of clinical trials in SCI has increased, the need for standardization of this examination has been emphasized in the literature $[8,9]$. Concomitantly, multiple educational vehicles $[10,11]$ have been developed to train practitioners in the performance of 
the examination. Moreover, the examination has been utilized to predict potential recovery in persons with SCIs.

Inconsistency in the description of the anorectal examination has existed since the introduction of the sacral sparing definition of incomplete injuries. The 1992 Standards booklet [7] states that "the external anal sphincter should be tested through insertion of the examiner's finger; perceived sensation should be graded as being present or absent". (ASIA, standards 1992, p 12) The accompanying Reference Manual to the Standards [12], published in 1994 indicated that during a digital rectal examination, "the patient is asked to describe any sensory awareness, including feelings of touch and/or pressure, when firm pressure with the digit is placed on the rectal walls". (Reference Manual 1994, p 14) This discrepancy is important because the innervation of the rectum is thought to be via the parasympathetic nervous system through S2-4 as compared to the innervation of the anal sphincter which is thought due to the inferior hemorrhoidal branches of the pudendal nerve to the sacral roots of S2-4 [13].

In 2011 [14] the decision was made to replace the term "deep anal sensation," which had been used since the inception of the examination, to "deep anal pressure." Although this change in terminology was reported as based upon consensus [15], the authors of this survey were confused by the words "deep" and "pressure," which seemed more appropriate to use for a rectal examination as compared to testing of the anal sphincter. In light of this confusion amongst senior practitioners performing the examination, we believed there could be inconsistency in the performance of the examination. This would subsequently change in what neurologic function was being tested, e.g., those performing a rectal exam testing autonomic function with those performing the anal examination testing somatic function and effect prognostication of recovery. Thus, we chose to perform an online survey of the perception, performance, and sources of knowledge of practitioners with regards to the anorectal component of the ISNCSCI exam to determine the need for further education and clarification regarding the examination.

\section{Methods}

A 10 question survey was developed by the authors to assess participants' knowledge, perceptions, and performance of the anorectal portion of the examination of persons with SCI, with particular attention to components of the ISNCSCI. As the study was considered primarily a means to determine educational needs, the authors concurred it did not meet institutional review board criteria.

Once the survey was completed, reviewed, and tested, it was forwarded to members of the International Spinal Cord
Society and members of other International Spinal Cord Organizations for distribution. The survey was also distributed to individual colleagues of the authors and these individuals were asked to distribute the survey to other practitioners in the field of SCI. Persons who chose to take the survey went online and provided their responses.

\section{Results}

Two hundred and fifteen responses were obtained to the questionnaire. Due to the nature of the survey distribution, we could not identify what percent of those who received a link to the survey actually chose to complete it. Of respondents, 157 were specialists in physical medicine and rehabilitation (PM\&R), 32 were physical therapists, 6 were neurologists, 6 were orthopedic surgeons, and 3 were neurosurgeons with the remaining respondents in other specialties. Questionnaires were returned from 100 practitioners in Europe, 82 individuals in North America, 12 in Australia/New Zealand, 5 in Asia, 3 in South America, and 3 in Africa, with the remaining respondents not reporting their country of origin. Forty-one percent of prescribers reported they had been performing the ISNCSCI exam for over 10 years.

Respondents were asked where they learned to perform the ISNCSCI exam. The majority $(n=110 ; 51 \%)$ reported they learned to perform the examination in their residency training while $24(11 \%)$ reported they learned it as part of a fellowship. Part of clinical education was listed as an education source by $73(34 \%)$ of participants and $79(37 \%)$ said they learned during on the job training. Thirty-five $(16 \%)$ had learned to perform the examination through the International Standards Training e-Learning Program (InSTeP), 48 (22\%) reported they were self-taught, 26 (12.1\%) learned to perform the exam as part of a clinical trial, and 2 reported they did not remember.

Respondents were asked "What does deep anorectal pressure mean to you?" 190 practitioners responded to the question and of these, 29 indicated pressure against the anal sphincter, 31 reported pressure applied to the rectum, and 130 reported pressure applied to the anal and rectal wall.

Respondents were also queried whether they personally performed the anal examination. Two did not respond to this question and $20(9 \%)$ of respondents said no. Further analysis of the data regarding the anorectal examination was limited to the 193 who reported that they personally performed the examination. Of the respondents who personally performed the exam, there were $150 \mathrm{PM} \& \mathrm{R}$ specialists, 20 physical therapists, 8 urologists, 6 orthopedic surgeons, 4 neurologists, 3 neurosurgeons, 1 pediatrician, and 1 neurogastroenterologist. Respondents were then asked what they did as part of their examination. Of these 193 respondents only 179 reported they did some type of 
penetrative anal examination; 39 (20\%) reported they routinely applied firm pressure against the anal sphincter, 56 (29\%) placed firm pressure against the rectal wall, and 84 $(43.5 \%)$ routinely applied pressure against the anal sphincter and the rectal wall. $153(79 \%)$ reported testing pinprick sensation against the junction of the internal and external skin, $170(88 \%)$ reported asking the patient to squeeze the examiner's finger, $106(55 \%)$ routinely determined the presence or absence of the bulbocavernosus reflex, and 93 (48\%) routinely checked the anal wink reflex.

With regards to the frequency of performing the examination, $71 \%$ of the respondents noted that they performed the examination a few times per month or more frequently. With regards to the sacral components of the autonomic standards, which are intimately connected to the rectal examination, $75 \%$ were aware of the autonomic standards but half of the respondents did not use them. Of the respondents that reported using the autonomic standards, 52 routinely used all three subcomponents, 13 routinely used only bladder, 7 routinely used bowel, and 1 routinely used sexual function.

Respondents were also queried about whether they would be amenable to performing the bulbocavernosus reflex as part of the ISNCSCI exam. Two hundred and nine participants answered this question and of these, 168 reported "Yes" while 41 reported "No."

\section{Discussion}

Our findings support our original hypothesis about the differing interpretation and inconsistency amongst practitioners with the terminology "deep anal pressure" and how to test for this. Nearly $80 \%$ of practitioners report stimulating the rectal wall when testing for anal sensation.

Anal sensation and rectal sensation follow different pathways and are processed differently in the brain. Using functional magnetic resonance imaging, Eickhoff et al. [16] demonstrated that anal stimulation activated the secondary somatosensory cortex, while rectal stimulation did not, instead activating a more anterior location on the precentral operculum. Distal pudendal nerve blockade will blunt anal sensation but not distal rectal sensation [17]. For the other aspects of the sensory portion of the ISNCSCI examination, the practitioner is assessing somatic function and not autonomic function; why the assessment of anorectal function would be different than other areas is unclear. Moreover, this issue of eliciting somatic vs. autonomic sensation during the anorectal examination has been identified as a possible source of concern in multiple reports $[8$, $18,19]$ and was even considered part of the reason for the change in terminology to "deep anal pressure [15]." Our survey did not ask about change in understanding of the term "deep anal pressure" over time and cannot determine whether the change in terminology has led to better or worse agreement of its meaning.

In detailed review of the training documents and original articles with regards to the assessment, it was determined that the Reference Manual for the International Standards for Neurological Classification of Spinal Cord Injury [10] continued the wording of the 1994 reference manual, "The patient is asked to describe any sensory awareness ... when firm pressure with the digit is placed on the rectal walls." This is in contrast to how practitioners were trained in the 1980's and 1990's, and the description in the booklet. Subsequent to this, the terminology "anorectal" was also used to describe the examination in the InSTeP examination [11]. Moreover, researchers have included the rectal component of the examination during recent studies when they have compared testing of S4-5 and anal sensation [3, 20, 21].

Another concern about this discrepancy in technique of the anorectal examination is that the ISNCSCI examination is often considered a standard component of SCI clinical trials, with a change in the American Spinal Injury Association Impairment Scale (AIS) grade as a primary outcome measure. In light of the findings of this study, it is quite possible that, despite attempts at standardization of training activities, different examiners are performing the anal examination in different ways. A recent clinical trial in acute SCI had a large percentage of subjects in the control group convert from complete to incomplete status based solely on anorectal sensation [22]. Whether this was due to a different examination technique at follow up or a true change is unclear.

Sacral sparing has been tested multiple times with regards to its usefulness for prediction of motor recovery after SCI. A recent report advocated for the use of S3 pressure sensation as compared to deep anal pressure as an alternative to the current neurologic examination [8]. A large study also reported on the usefulness of sacral sparing with regards to prediction of ambulation finding that only voluntary anal contraction and the S4-5 light touch and pinprick scores contributed to the ability to predict independent ambulation [18]. On the other hand, compared with persons with complete SCI (AIS A), persons who had sacral sparing (AIS B) at rehabilitation discharge reported greater functional independence, better health and greater social participation at 1 year postinjury [23].

The assessment of anal or rectal sensation has usefulness for the prediction of bladder function [24, 25] and sexual function $[26,27]$ when performed in conjunction with the bulbocavernosus reflex. The bulbocavernosus is performed by squeezing the penis or clitoris at the same time as the examiner's finger is in the anus so that they can feel an anal contraction. Alternatively, it can be elicited via tug of the catheter in conjunction with the examiner's finger in the anus. It is possible the bulbocavernosus could be predictive 
of retention of other autonomic functions. According to this survey, $50 \%$ percent of practitioners are already performing the bulbocavernosus reflex; however, it is not part of the routine ISNCSCI examination. Based upon the strong interest of patient's in recovery of autonomic function [28], consideration could be given to making this a routine part of the ISNCSCI examination.

It may be useful to have two alternate pathways to document recovery of neurologic function which could be based upon the initial level of injury or the purpose of the examination-one inclusive of the anorectal examination with documentation of both the anal and rectal examinations with a goal of predicting and evaluating completeness of sacral autonomic function and the other without performance of the anal examination with a goal of prediction of recovery of motor function. Performance of a digital rectal examination is often inconvenient for the practitioner and the patient as it can stimulate a reflex bowel movement and having an option for an alternative means of documenting a complete injury for purposes of studying somatic sensory and motor function would be useful. Concomitantly, the inconsistency with the performance of the anorectal examination and the lack of performance of reflexes documents the need for greater evaluation of remaining autonomic function after SCI. As persons with SCIs are interested in more than just walking, it is imperative that scientists and clinicians have the appropriate tools and utilize standardized techniques such as the International Standards to document remaining Autonomic Function after Spinal Cord Injury (ISAFSCI) [29, 30] to document recovery of not only motor function but also the autonomic functions that are under partial volitional control, those of bladder, bowel, and sexual function, and the remaining autonomic functions.

This brief survey documents the need for further discussion of the evolution of the ISNCSCI examination and training of professionals. More research is needed to find alternatives to the anorectal examination to determine a sensorimotor incomplete injury. In order to determine the significance of rectal vs. anal sensation on recovery of somatic and autonomic functions, reliable methods for bedside testing of each are needed.

\section{Compliance with ethical standards}

Conflict of interest MA is a consultant for in-vivo therapeutics. The remaining authors declare that they have no competing interests.

\section{References}

1 Krishna V, Andrews H, Varma A, Mintzer J, Kindy MS, Guest J. Spinal cord injury: How can we improve the classification and quantification of its severity and prognosis. J. Neurotrauma. 2014;31:215-27.
2 Zariffa J, Kramer J, Jones L, Lammertse D, Curt A. European multicenter study about SCI Group, et al. sacral sparing in SCI; beyond the S4-S5 and anorectal examination. The Spine 2012;12:389-400.

3 Samdani A, Chafetz RS, Vogel LC, Betz RR, Gaughan JP, Mulcahey MJ. The international standards for neurological classification of spinal cord injury: relationship between S4-5 dermatome testing and anorectal testing. Spinal. Cord. 2011;49:352-6.

4 American Spinal Injury Association. International Standards for the Neurological Classification of Spinal Cord Injury. Chicago: American SpinalInjury Association; 2003.

5 American Spinal Injury Association. Standards for Neurological Classification of Spinal Cord Injury Patients. Chicago: American Spinal Injury Association; 1989.

6 American Spinal Injury Association. Standards for Neurological Classification of Spinal Cord Injury Patients. Chicago: American Spinal Injury Association; 1982.

7 American Spinal Injury Association/International Medical Society of Paraplegia (ASIA/IMSOP). International Standards for Neurological and Functional Classification of Spinal Cord Injury Patients (revised). Chicago: American Spinal Injury Association; 1992.

8 Marino RM, Schmidt-Read M, Kirshblum S, Dyson-Hudson TA, Tansey K, Morse LR, et al. Reliability and validity of S3 pressure sensation as an alternative to deep anal pressure in neurologic classification of persons with spinal cord injury. Arch. Phys. Med. Rehabil. 2016;97:1642-6.

9 Mulcahey MJ, Gaughan J, Betz RB, Vogel LC. Rater agreement on the ISCSCI motor and sensory scores obtained before and after formal training in testing technique. J. Spinal. Cord. Med. 2007;30: S146-9.

10 American Spinal Injury Association. Reference Manual for the International Standards for Neurological Classification of Spinal Cord Injury. Chicago: American SpinalInjury Association; 2003.

11 www.asia-spinalinjury.org/elearning. Accessed June 21, 2017.

12 American Spinal Injury Association. Reference Manual for the International Standards for Neurological Classification of Spinal Cord Injury. Chicago: American SpinalInjury Association; 1994.

13 Rogers J. Testing for and the role of anal and rectal sensation. Bailliers Clin Gastroenterol 1992;6:179-191.

14 Kirshblum SC, Burns S, Biering-Sorensen F, Donovan W, Graves $\mathrm{D}$, Jha $\mathrm{A}$, et al. International standards for neurological classification of spinal cord injury (Revised 2011). J. Spinal. Cord. Med. 2011;34:346-52.

15 Kirshblum S, Waring W. Updates for the international standards for neurological classification of spinal cord injury. Phys. Med. Rehabil. Clin. N. Am. 2014;25:505-17.

16 Eickhoff SB, Lotze M, Wietek B, Amunts K, Enck P, Zilles K. Segregation of visceral and somatosensory afferents: an fMRI and cytoarchitectonic mapping study. Neuroimage. 2006;31: 1004-1014.

17 Chan CLH, Ponsford S, Scott SM, Swash M, Lunniss PJ. Contribution of the pudendal nerve to sensation of the distal rectum. Br. J. Surg. 2005;92:859-865.

18 van Middendorp JJ, Hosman AJF, Pouw MH, Van de Meent H. Is determination between complete and incomplete traumatic spinal cord injury clinically relevant? Validation of the ASIA sacral sparing criteria in a prospective cohort of 432 patients. Spinal. Cord. 2009;47:809-816.

19 Wietek BM, Baron CH, Erb M, Hinninghofen H, Baktke A, Kaps $\mathrm{H}$, et al. Cortical processing of residual ano-rectal sensation in patients with spinal cord injury: an fMRI study. Neurogastroenterol. Motil. 2008;20:488-497.

20 Krisa L, Middleton D, Faro S, Calhoun C, Mohamed F, Mulcahey M. Cerebral activation during the test of spinal cord injury severity 
in children; an fMRI methodological study. Top Spinal Cord Injury Rehabil 2013;19:121-8.

21 Vogel L, Samdani A, Chafetz R, Gaughan J, Betz R, Mulcahey MJ. Intra-rater agreement of the anorectal exam and classification of injury severity in children with spinal cord injury. Spinal. Cord. 2009;47:687-691.

22 Lammertse DP, Jones LA, Charlifue SB, Kirshblum SC, Apple $\mathrm{DF}$, Ragnarsson KT, et al. Autologous incubated macrophage therapy in acute, complete spinal cord injury: results of the phase 2 randomized controlled multicenter trial. Spinal. Cord. 2012;50:661-671.

23 Kirshblum S, Botticello A, Lammertse DP, Marino RJ, Chiodo AE, Jha A. The impact of sacral sensory sparing in motor complete spinal cord injury. Arch. Phys. Med. Rehabil. 2011;92:376-383.

24 Alexander MS, Carr C, Chen YY, McLain A. The use of the neurologic examination to predict awareness of lower urinary tract function post SCI. Spinal. Cord. 2017;55:795, https://doi.org/10. 1038/sc2017.55.
25 Weiss DJ, Fried GW, Chancellor MB, Herbison GH, Ditunno JF, Staas WE. Spinal cord injury and bladder recovery. Arch. Phys. Med. Rehabil. 1996;77:1133-5.

26 Sipski ML, Alexander CJ, Rosen RC. Sexual arousal and orgasm in women: effects of spinal cord injury. Ann. Neurol. 2001; 49:35-44.

27 Sipski M, Alexander CJ, Gomez-Marin O. Effects of level and degree of spinal cord injury on male orgasm. Spinal. Cord. 2006;44:798-804.

28 Anderson KD. Targeting Recovery: Priorities of the Spinal CordInjured Population. J. Neurotrauma. 2004;21:1371-83.

29 Alexander MS, Biering-Sorensen F, Bodner D, Brackett NL, Cardenas D, Charlifue S, et al. International standards to document remaining autonomic function after spinal cord injury. Spinal. Cord. 2009;47:36-43.

30 Krassioukov A, Biering-Sorensen F, Donovan W, Kennelly M, Kirshblum S, Krogh K, et al. International standards to document remaining autonomic function after spinal CordInjury (ISAFSCI). Top SCI Rehabil 2012;18:283-96. First Edition 2012 